\title{
New results from CERES
}

\section{A. Marín for the CERES Collaboration $\dagger$}

\begin{abstract}
.
During the year 2000 the CERES experiment, upgraded with a radial drift TPC, took a large data sample of $\mathrm{Pb}$ on $\mathrm{Au}$ collisions at $158 \mathrm{AGeV}$ triggered on the $8 \%$ most central collisions. A very detailed calibration of the radial drift TPC was since completed. Preliminary results on $e^{+} e^{-}$pairs and $\phi$ mesons reconstructed in the $K^{+} K^{-}$channel are presented.
\end{abstract}

\section{Introduction}

QCD calculations predict a transition from ordinary hadronic matter into a plasma of deconfined quarks and gluons at high energy density. Dileptons, which have negligible final state interactions, represent a very suitable probe for the study of this new state of matter. The CERES/NA45 experiment at the CERN SPS has made major contributions to the measurement of low-mass $\mathrm{e}^{+} \mathrm{e}^{-}$pairs in ultra-relativistic heavyion collisions. CERES has measured an enhanced dilepton production in the invariant mass region $m_{e^{+} e^{-}}>0.2 \mathrm{GeV} / \mathrm{c}^{2}$ in $\mathrm{S}+\mathrm{Au}$ at $200 \mathrm{AGeV}$ [1] and in $\mathrm{Pb}+\mathrm{Au}$ at $158 \mathrm{AGeV}[2$ and at $40 \mathrm{AGeV}$ [3] compared to the contribution from known hadronic sources. The enhancement is absent in p-induced reactions [4. Pion annihilation has been taken into account as an additional mechanism for $\mathrm{e}^{+} \mathrm{e}^{-}$production but the experimental spectra cannot be explained without introducing medium modifications of vector mesons, particularly of the $\rho$. The question of possible modifications of other vector mesons, specially of the $\omega$ and the $\phi$, and the role of chiral symmetry restoration remained however open.

In order to further investigate the enhancement, the CERES spectrometer was upgraded during 1998 by the addition of a Time Projection Chamber (TPC) with radial electric drift field [5, 6, 7] which improves the mass resolution and the electron identification. During the year 2000 CERES took a large data sample consisting of $30 \cdot 10^{6}$ and $3 \cdot 10^{6}$ events of $\mathrm{Pb}$ on $\mathrm{Au}$ collisions at $158 \mathrm{AGeV}$ triggered on the $8 \%$ and $20 \%$ most central collisions, respectively. The challenge was to calibrate the highly complex radial drift TPC to bring the momentum resolution to the design limit.

\section{Experimental Setup}

The CERES experiment (Fig. 1) is optimized to measure low mass electron pairs close to mid-rapidity $(2.1<\eta<2.6)$ with full azimuthal coverage. A vertex telescope, composed of two Silicon Drift Detectors (SDD) positioned at $10 \mathrm{~cm}$ and $13.8 \mathrm{~cm}$

$\dagger$ For the full CERES Collaboration author list and acknowledgments, see Appendix "Collaborations" of this volume. 


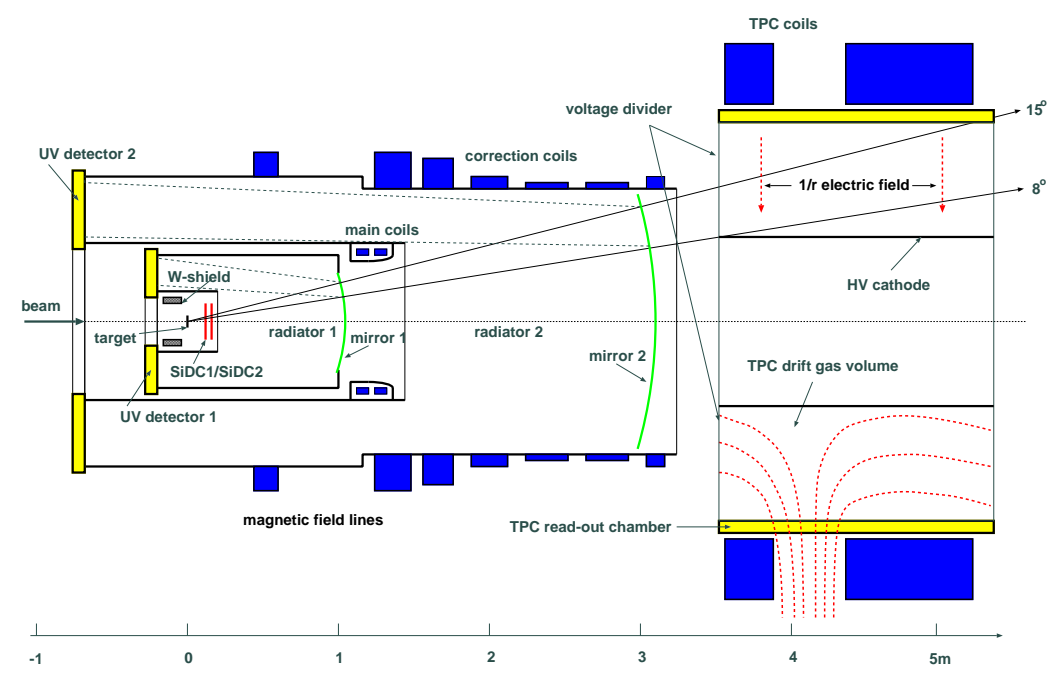

Figure 1. Cross section through the upgraded setup of the CERES spectrometer

downstream of a segmented $\mathrm{Au}$ target, provides a precise vertex reconstruction, angle measurement for charged particles and rejection of close pairs from $\gamma$ conversions and $\pi^{0}$ Dalitz decays. Two Ring Imaging CHerenkov (RICH) detectors, operated at a high threshold $\left(\gamma_{t h}=32\right)$, are used for electron identification in a large hadronic background. The new radial-drift TPC, positioned downstream of the original spectrometer, has an active length of $2 \mathrm{~m}$ and a diameter of $2.6 \mathrm{~m}$. A gas mixture of $\mathrm{Ne}(80 \%)$ and $\mathrm{CO}_{2}(20 \%)$ is used. It is operated inside a magnetic field $(\vec{B}$, indicated by the dashed field lines inside the TPC in Fig. (1) with a maximal radial component of $0.5 \mathrm{~T}$ and provides up to 20 space points for each charged particle track. This is sufficient for the momentum determination and for additional electron identification via $\mathrm{d} E / \mathrm{d} x$ in the TPC. In the configuration with the TPC, the magnetic field between the two RICH detectors is switched off and there is thus no deflection between them, allowing to use them in a combined mode resulting in an increased electron efficiency. Moreover, the TPC opens new possibilities to study hadronic observables [8, 9, 10, 11, 12].

\section{Calibration of the TPC}

One of the main tasks of the TPC calibration was to understand the electric field $(\vec{E})$ which is dominantly radial with $E_{r} \sim 1 / r$ but has a small azimuthal component due to the polygonal shape of the TPC and small longitudinal components at the end caps. A custom program based on the relaxation method was developed for calculating the electric field including the proper knowledge of the field cage resistors, the field distortions caused by slightly displaced chambers and the leakage of the amplification field through the cathode wire plane.

The magnetic field in the TPC is generated by two coils running with opposite currents (Fig (1). The two main components $B_{z}$ and $B_{r}$ change with $r$ and $z$. A field map was measured before the installation of the TPC in the experimental area. Compared to the nominal magnetic field calculated with the POISSON program the measured field deviates from azimuthal symmetry by a few \%. These deviations are now included as corrections to the nominal field map. 
The electron mobility as a function of $\vec{E}$ was determined from laser events containing tracks at known positions for a given set of gas parameters. Relative run-by-run variations caused by changes in composition, temperature, and pressure (monitored by the slow control system of the experiment) are calculated with the MAGBOLTZ program [13. The transformation from pad, time, and plane to the laboratory coordinate system $(x, y$, and $z$ ) is done using a fourth-order Runge-Kutta method. The drift trajectory is calculated starting at the cathode plane using in each point the drift velocity vector, $\vec{v}_{D}=\mu \frac{1}{1+(\mu|B|)^{2}}\left(\vec{E}+\mu(\vec{E} \times \vec{B})+\mu^{2}(\vec{E} \cdot \vec{B}) \vec{B}\right)$ where the components parallel to the electric field and parallel to $(\vec{E} \times \vec{B})$ have been modified to account for the difference observed between using $\vec{v}_{D}$ from the above equation and the Monte Carlo drift option in the MAGBOLTZ program [13. To fully account for the Lorentz angle a correction is extracted from the symmetry of the $1 / \mathrm{p}$ distribution of identified pions under the assumption that very high momentum particles have vanishing curvature. After all these corrections the local distortions are small compared to the resolution.

The momentum of tracks from the target is determined from the combination (resolution-weighted) of a 3-parameter fit that takes into account multiple scattering before the TPC (dominant for low-momentum particles) and a 2-parameter fit that assumes that tracks come from the vertex (dominant for high-momentum particles). The momentum resolution depends on the number of hits on a track and on the singlehit position resolution. New hit finding and track finding algorithms were developed
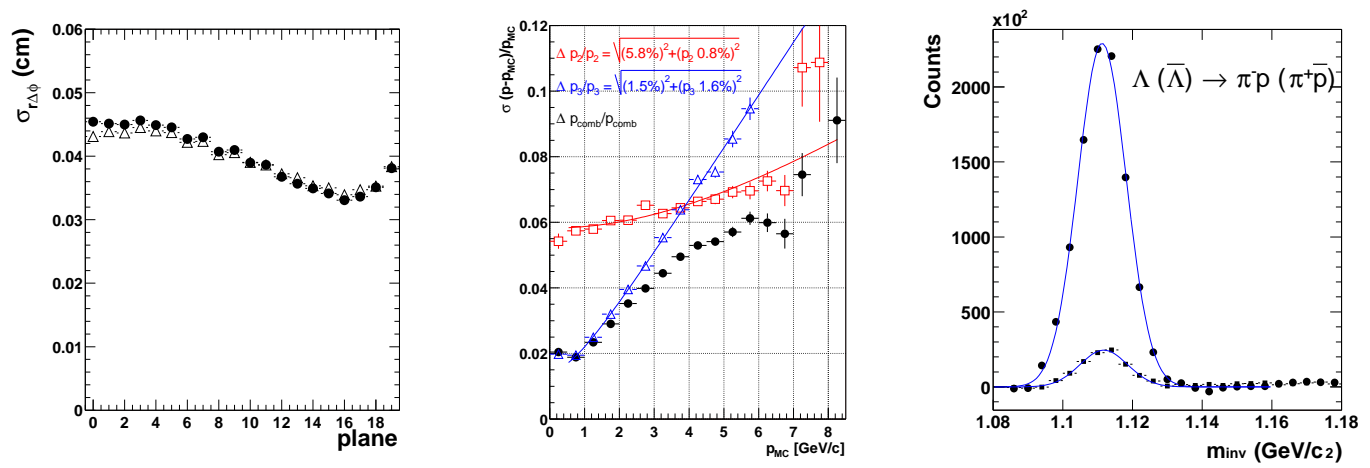

Figure 2. (Left) Single-hit position resolution in azimuthal direction ( $\mathrm{r} \Delta \phi)$ as function of the plane in the TPC $(z)$ for events triggered on the $8 \%$ (circles) and $20 \%$ (triangles) most central collisions obtained from hits on tracks with a momentum $p>1 \mathrm{GeV} / \mathrm{c}$. (Middle) Momentum resolution from a simulation with single hit position resolution as in the data for the 3 parameter fit (triangles), for the 2 parameter fit (squares) and for the combined fit (circles). (Right) Invariant mass spectra of $\mathrm{p} \pi^{-}\left(\bar{p} \pi^{+}\right)$pairs after background subtraction for $2.0<y_{\Lambda}<2.4$ and $p_{t}^{\Lambda}>1.5 \mathrm{GeV} / \mathrm{c}$. After fitting the peaks of $\Lambda$ (circles) and $\bar{\Lambda}$ (squares) with a Gaussian function widths of $6.94 \pm 0.02 \mathrm{MeV} / \mathrm{c}^{2}$ and $6.85 \pm 0.13 \mathrm{MeV} / \mathrm{c}^{2}$ are obtained. The measured $\bar{\Lambda} / \Lambda$ ratio is $0.105 \pm 0.002$.

to better resolve close hits and to improve tracking efficiency at very low momenta. Tracks above $1 \mathrm{GeV} / \mathrm{c}$ momentum are found with an efficiency of $90 \%$ due to non instrumented areas and have an average number of hits of 18.7. Down to a momentum of $0.3 \mathrm{GeV} / \mathrm{c}$ the efficiency is still $80 \%$ and the average number of hits is 17.2 . The single-hit position resolution is obtained comparing the reconstructed hit positions 
with the ideal hit positions given by the fitted trajectory of a track. The width of the residual distribution gives the spatial resolution. The resolution in azimuthal direction scaled with the radius $r$ of the hit $\left(\sigma_{r \Delta \phi}\right)$ is shown in Fig. 2. It deteriorates slightly with increasing hit multiplicity in the TPC. The momentum resolution has also been evaluated using a microscopic drift Monte Carlo simulation with singlepoint resolution as observed in the data (Fig. (2). It translates into a mass resolution of about $4 \%$ at the $\phi$ meson mass reconstructed in the $e^{+} e^{-}$channel. The momentum resolution was checked in the data by looking at the width of the reconstructed $\Lambda$ and $K_{S}^{0}$ in the invariant-mass spectrum (Fig. 22). The detailed calibration improves the mass resolution by at least a factor 1.7 compared to the old calibration [14.
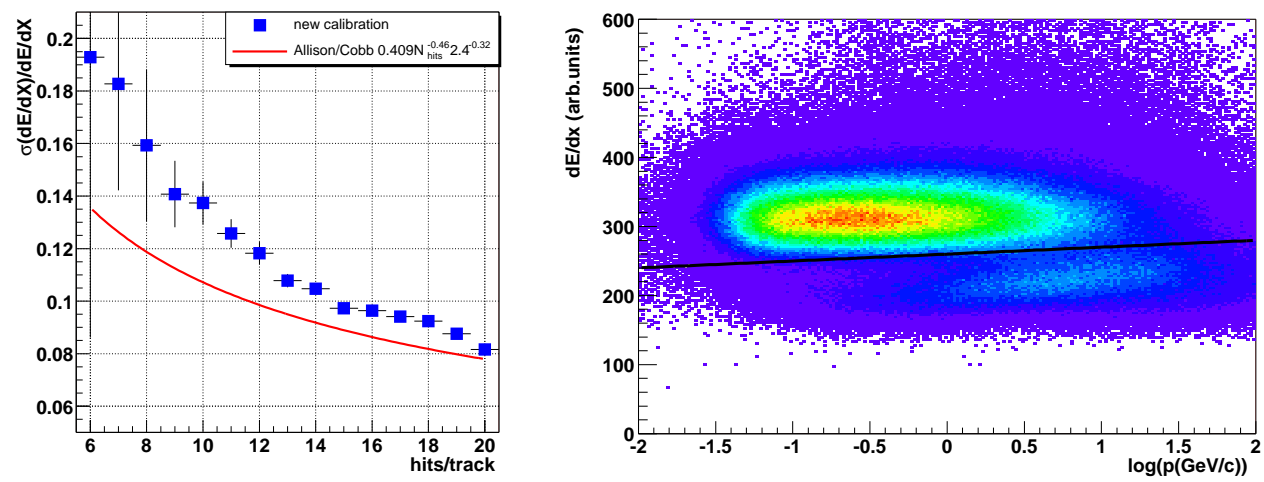

Figure 3. (Left) $\mathrm{d} E / \mathrm{dx}$ resolution of electrons as a function of number of hits in the track after the new calibration (squares) compared to the parameterization of Allison/Cobb (line). (Right) $\mathrm{d} E / \mathrm{dx}$ signal in the TPC versus momentum for electrons as selected with the RICH detector. The inclined cut against pions (black line) show the additional rejection power from the TPC.

The pad-to-pad gain calibration is obtained from the total hit amplitude in the pad of maximum amplitude. A correction of the undershoot after each pulse obtained from non-zero suppressed data was included [15]. An electron attachment correction is determined by analysing the dependence of the hit amplitude on the drift length and taking into account the different particle abundancies for each polar angle $\theta$. The $\mathrm{d} E / \mathrm{dx}$ of a track is calculated as the truncated mean of the individual hit amplitudes. For the maximum number of hits on tracks the $\mathrm{d} E / \mathrm{dx}$ resolution approaches the parameterization by Allison and Cobb (Fig. Bi), which implies that most of the electrons in this analysis are measured with $\mathrm{d} E / \mathrm{dx}$ resolution of better than $10 \%$.

\section{Electron analysis}

The main difficulties of the electron analysis are the low probability of electromagnetic decays and the large amount of combinatorial background from $\gamma$ conversions and Dalitz decays. To select electrons among all charged hadrons the RICH detectors and the $\mathrm{d} E / \mathrm{dx}$ signal in the TPC are used. The analysis chain provides track segments in the different detectors. The two RICH detectors are used in a combined mode in this analysis. Rings with asymptotic radius and a (double Hough) amplitude above a given threshold for the full ring acceptance (slowly decreasing threshold towards the edges) 
are taken as RICH track segments. With these cuts a pion rejection of about 0.999 is achieved for an electron efficiency of about 0.55 . Quality cuts (matching between SDD1/2 of $2 \mathrm{mrad}$ and at least 12 hits in the TPC track segments) are applied.

Global tracks are constructed by combining track segments in SDD, TPC and RICH with a $2 \sigma$ momentum-dependent matching window. The electron $\mathrm{d} E / \mathrm{dx}$ signal in the TPC is independent of momentum (Fig. B). A primary electron selection is done based on the $\mathrm{d} E / \mathrm{dx}$ resolution. Finally a more restricted cut to further suppress pions is applied (Fig. 3i).

Very good electron identification is not enough. Electron pairs from $\gamma$ conversions and $\pi^{0}$ Dalitz decays need to be identified by topology and removed from the sample to reduce combinatorial background. They are characterized by their small opening angle and low momentum. As the detectors have a finite two-track resolution the most effective way of rejecting conversions in the target and close Dalitz pairs is by rejecting tracks with at least twice the minimum ionizing energy loss signal in both SDD's where all hit amplitudes have been resummed within $7 \mathrm{mrad}$ around the each SDD track segment. Low-amplitude tails in the SDD $\mathrm{d} E / \mathrm{dx}$ distribution are also removed. Late conversions (mostly in SDD1) are removed by an upper cut in each RICH ring amplitude and by a cut in the distance to another TPC track of opposite sign and electron $\mathrm{d} E / \mathrm{dx}$. To reduce $\pi^{0}$ Dalitz decay contributions only electron tracks with no opposite charge electron track within $35 \mathrm{mrad}$ are taken for analysis. Finally, only electron tracks in the geometrical acceptance $0.14 \mathrm{rad}<\theta<0.243 \mathrm{rad}$ and with a $p_{t}>0.2 \mathrm{GeV} / \mathrm{c}$ are selected.

The invariant-mass distributions of unlike-sign pairs and of like-sign pairs that is a measure of the combinatorial background after full rejection are shown in Fig. 4 In order to reduce the statistical errors an unlike-sign combinatorial background using the mixedevent technique has also been evaluated. For masses below $0.2 \mathrm{GeV} / \mathrm{c}^{2}$, the

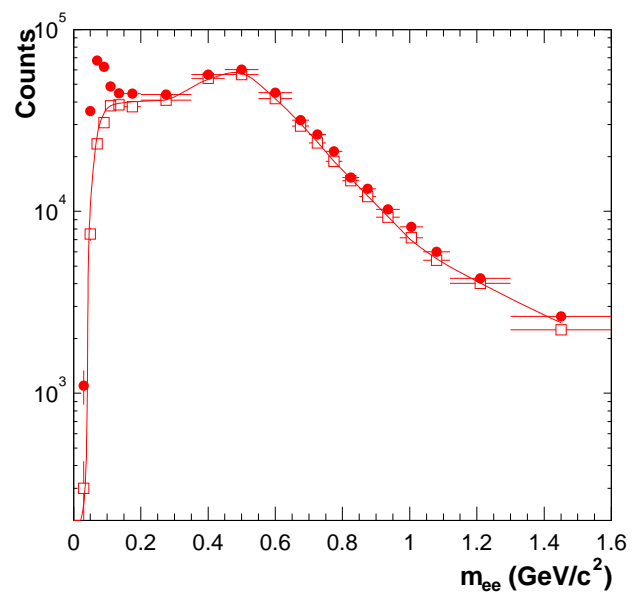
mixed-event background deviates from the like-sign background due to ring reconstruction effects; the mixed-event background is normalized to the likesign pair background in the mass region $0.2 \mathrm{GeV} / \mathrm{c}^{2}<\mathrm{m}_{e^{+} e^{-}}<0.6 \mathrm{GeV} / \mathrm{c}^{2}$. The physics signal is obtained by subtracting the like-sign pairs or the mixed event background from the unlike-sign pairs as $N_{e^{+} e^{-}}-\left(N_{e^{+} e^{+}}+N_{e^{-} e^{-}}\right)$. This analysis is based on about $18 \cdot 10^{6}$ events corresponding aproximately to $80 \%$ of the events where both RICH detectors were operational.

Figure 4. Invariant-mass distribution of unlike-sign pairs (full symbols), like-sign pairs (open symbols), and mixedevent background (line) normalized to like-sign pairs background after full rejection. 


\section{Low-mass $\mathbf{e}^{+} \mathbf{e}^{-}$results}

The preliminary $\mathrm{e}^{+} \mathrm{e}^{-}$invariant-mass spectrum normalized to the $\pi^{0}$ Dalitz peak of the hadronic decay cocktail is shown in Fig. [5] The hadron decay cocktail 17 has been folded with the experimental momentum resolution and energy loss due to bremsstrahlung. Acceptance, opening-angle, and transverse-momentum cuts are applied. An excess of pairs for $\mathrm{m}_{e^{+} e^{-}}>0.2 \mathrm{GeV} / c^{2}$ is visible. Using the like-
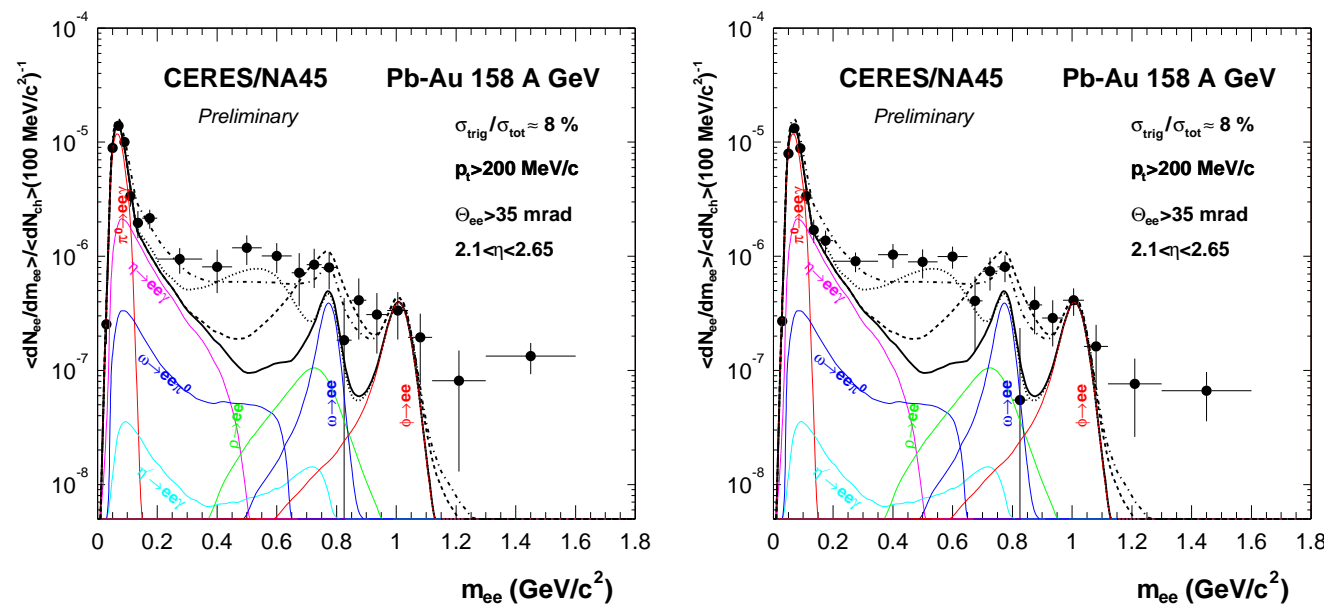

Figure 5. Invariant-mass spectrum of $e^{+} e^{-}$-pairs normalized to the expectation from the hadron decay cocktail (thick solid line) in the $\pi^{0}$ Dalitz peak using the like-sign background (left) or the mixed-event background (right). The expectations from model calculations assuming the vacuum $\rho$ spectral function (dashed), a dropping mass (dotted) or a in medium spread $\rho$ width (dasheddotted) are also shown.

sign background, the number of pairs in the Dalitz region $\left(\mathrm{m}_{e^{+} e^{-}}<0.2 \mathrm{GeV} / \mathrm{c}^{2}\right)$ is $2826 \pm 114$ with a signal to background ratio (S/B) of $1 / 1.78$. Using the mixedevent background, the number of open pairs $\left(\mathrm{m}_{e^{+} e^{-}}>0.2 \mathrm{GeV} / \mathrm{c}^{2}\right)$ is $2023 \pm 182$ with a S/B of 1/14.6. The enhancement factor for $0.2 \mathrm{GeV} / \mathrm{c}^{2}<\mathrm{m}_{e^{+} e^{-}}<1.1 \mathrm{GeV} / \mathrm{c}^{2}$ compared to the hadron decay cocktail is $3.1 \pm 0.3$ (stat). Further optimization of cuts based on Monte Carlo simulations is in progress and is expected to improve the statistical errors.

The experimental results are compared to theoretical models based on hadronic decays and $\pi^{+} \pi^{-}$annihilation. The $\rho$-propagator is treated [18] in 3 ways: vacuum $\rho$, modifications following Brown-Rho scaling [19, and modifications via $\rho$-hadron interactions 20. The data clearly disfavour the vacuum $\rho$. In the region between the $\omega$ and the $\phi$ the data seem to favour the many-body approach over Brown-Rho scaling. We have to await the final results of the analysis before a stronger statement can be made.

\section{The $\phi$ meson puzzle}

One of the open issues at the CERN SPS is the $\phi$ puzzle. Two experiments, one measuring the $\phi$ meson in the $\mu^{+} \mu^{-}$decay channel (NA50), and the other (NA49), measuring it in the $K^{+} K^{-}$channel, obtain yields that differ by factors between 2 and 
4 in the common $m_{t}$ range [21. Further, the $m_{t}$ spectra exhibit a different inverse slope parameter [21], 305 $\pm 15 \mathrm{MeV}$ in NA49 and $218 \pm 6 \mathrm{MeV}$ in NA50, fitted in their $m_{t}$ acceptance regions.
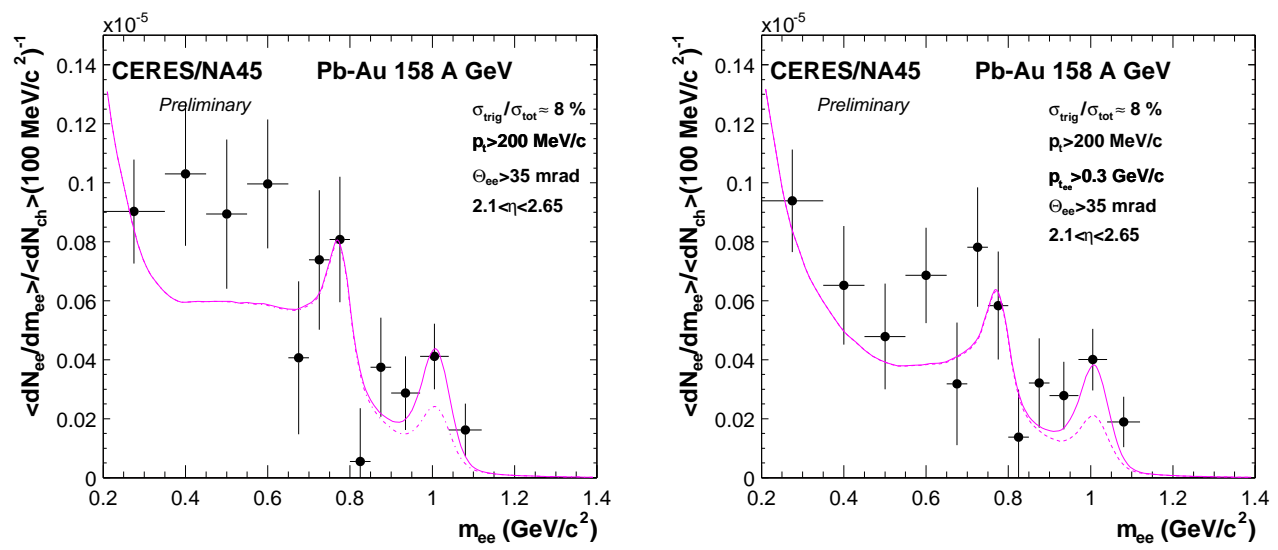

Figure 6. Invariant-mass spectrum of $e^{+} e^{-}$-pairs together with the expectations from model calculations assuming in-medium spread $\rho$ width plus the thermal $\phi$ yield (thin solid) or the thermal yield reduced to $50 \%$ (dashed) are shown. The left plot corresponds to the standard set of cuts and the right one has an additional electron pair $p_{t}$ cut of $0.3 \mathrm{GeV} / \mathrm{c}$.

We have compared our preliminary mass spectrum with a calculated spectrum based on a $\phi$-yield as predicted in a thermal model [22] which also describes the NA50 yield (left of Fig. 6). The lines also contain the $\rho$-contribution. Within a window of $\pm 1 \sigma$ around the $\phi$ peak the ratio of data to simulation is $1.1 \pm 0.3$. With an additional electron pair $p_{t}$ cut of $300 \mathrm{MeV} / \mathrm{c}$ (right of Fig. 6) the ratio is $1.2 \pm 0.3$. Obviously this is in very good agreement with our data. Reducing the $\phi$-yield to $50 \%$ of the above value (dashed lines of Fig. (6) leads to a ratio of $1.9 \pm 0.5$ and $2.1 \pm 0.6$. The reduced yield that would agree with the NA49 observation for $\phi \rightarrow K^{+} K^{-}$seems in disagreement with our preliminary data. The evaluation of absolute efficiencies and systematic errors are needed to draw a definitive conclusion.

The upgraded spectrometer offers the unique possibility to study simultaneously the $\phi$ meson in the $K^{+} K^{-}$decay channel. To study this channel, we assign to all charged particles the kaon mass (no particle identification). TPC tracks in the geometrical acceptance $0.14 \mathrm{rad}<\theta<0.24 \mathrm{rad}$ with at least 12 hits and with a transverse momentum $p_{t}>0.25 \mathrm{GeV} / \mathrm{c}$ that are matched to a SDD track within $2.5 \sigma$ momentum-dependent matching window are used. The combinatorial background is calculated using the mixed-event technique. An opening-angle cut of $0.015 \mathrm{rad}<\theta_{12}<0.17 \mathrm{rad}$ is applied to improve the signal-to-background ratio. A cut $q_{t}<0.19-0.4 \cdot \alpha^{4}-0.4 \cdot \alpha^{2}$, where $\alpha$ is the Podolanski-Armenteros parameter $\alpha=\left(p_{L}^{+}-p_{L}^{-}\right) /\left(p_{L}^{+}+p_{L}^{-}\right)$and $p_{L}^{ \pm}\left(q_{t}\right)$ is the decay track momentum component parallel (transverse) to the $\phi$ momentum in the laboratory frame, is also applied to avoid contamination from other particles species. The signal is studied for $p_{t}^{\phi}>1$ $\mathrm{GeV} / \mathrm{c}$ and rapidity $2.0<y^{\phi}<2.4$. Acceptance and pair reconstruction efficiency corrections are obtained from GEANT simulations where $\phi$ decays with a distribution in transverse momentum $d N / d p_{t}=C \cdot p_{t} \cdot \exp \left(-m_{t} / T\right)$ and a Gaussian rapidity distribution with a standard deviation of 1.2 are embedded into the background of 
real events. The preliminary invariant mass spectrum after background subtraction
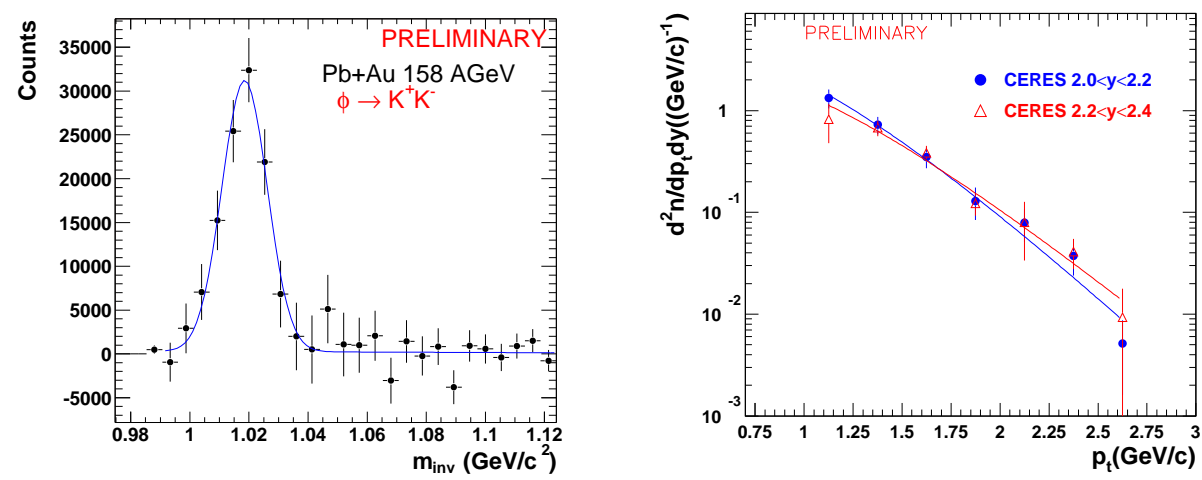

Figure 7. Preliminary invariant-mass spectrum of $K^{+} K^{-}$pairs after background subtraction for $2.2<y_{\phi}<2.4$ and $1.5 \mathrm{GeV} / \mathrm{c}<p_{t}^{\phi}<1.75 \mathrm{GeV} / \mathrm{c}$. A peak corresponding to the $\phi$ meson is observed (left). Transverse momentum spectrum of the $\phi$ meson corrected for acceptance and efficiency. Also shown are fits with a mentioned distribution. For fits see text.

and the transverse momentum spectra corrected for efficiency and acceptance are presented in Fig. 7 The values of the inverse slope parameter obtained after fitting with the function given above are $\mathrm{T}=218 \pm 12 \mathrm{MeV}$ and $\mathrm{T}=246 \pm 22 \mathrm{MeV}$ for the rapidity intervals $2.0<\mathrm{y}<2.2$ and $2.2<\mathrm{y}<2.4$, respectively. Evaluation of systematic errors and of the exact centrality necessary to compare to other experiments are in progress.

\section{References}

[1] G. Agakichiev et al. CERES Collaboration Phys. Rev. Lett. 75 (1995) 1272.

[2] G. Agakichiev et al. CERES Collaboration Phys. Lett. B 422 (1998) 405; B. Lenkeit for the CERES Collaboration Nucl. Phys. A 661 (1999) 23c. J.P. Wessels for the CERES Collaboration Nucl. Phys. A 715 (2003) 607c.

[3] D. Adamova et al. Phys. Rev. Lett. CERES Collaboration 91 (2003) 042301.

[4] G. Agakichiev et al. CERES Collaboration Eur. Phys. Jour. C 4 (1998) 231.

[5] Addendum to proposal SPSLC/P280: CERN/SPSLC 96-35/P280 Add.1.

[6] Technical Note on the NA45/CERES upgrade. CERN/SPSLC 96-50 (1996).

[7] A. Marín for the CERES Collaboration Nucl. Phys. A 661 (1999) 673c.

[8] H. Appelshäuser for the CERES Collaboration Nucl. Phys. A698 (2002) 253c.

[9] D. Adamova et al. CERES Collaboration Nucl. Phys. A714 (2003) 124.

[10] D. Adamova et al. CERES Collaboration Phys. Rev. Lett. 90 (2003) 022301.

[11] D. Adamova et al. CERES Collaboration Nucl. Phys.A 727 (2003) 97.

[12] H. Sako CERES Collaboration, these proceedings.

[13] R. Veenhof, Nucl. Instr. and Meth. 419 (1998) 726; http://consult.cern.ch/writeup/garfield/ S.F. Biagi, Nucl. Instr. and Meth. 421 (1999) 234.

[14] W. Schmitz for the CERES Collaboration Jour. Phys. G 28 (2002) 1861.

[15] L. Dietrich, Diploma Thesis, University of Heidelberg (2001).

[16] W.W.M. Allison and J.H. Cobb Ann. Rev. Nucl. Part. Sci. 30 (1980) 253.

[17] H. Sako for the CERES Collaboration. Technical Report 03-25 (2000).

[18] R. Rapp, private comunication.

[19] G.E. Brown and M. Rho. Phys. Rep. 363 (2002) 85.

[20] R. Rapp and J. Wambach, Adv. Nucl. Phys. 25 (2000) 1; private comunication.

[21] D. Röhrich J. Phys. G 27 (2001) 355.

[22] P. Braun-Munzinger, I. Heppe and J. Stachel Phys. Lett.B 465 (1999) 15. 